\title{
Charge Storage Mechanisms of Single Layer Graphene in Ionic Liquid
}

Jianglin Ye, ${ }^{+,+, s}$ Yih-Chyng Wu, ${ }^{\ddagger}, \|, \mathbb{S}$ Kui Xu, ${ }^{,, \|}$Kun Ni, ${ }^{+} \mathrm{Na}$ Shu, ${ }^{+}$Pierre-Louis Taberna, ${ }^{\ddagger}, \|$ Yanwu Zhu, ${ }^{*,+}$ and Patrice Simon, ${ }^{*, \neq, \|}$

${ }^{\dagger}$ Hefei National Research Center for Physical Sciences at the Microscale, \& CAS Key Laboratory of Materials for Energy Conversion, \& Department of Materials Science and Engineering, University of Science and Technology of China, Hefei, Anhui 230026, P. R. China

‡ Université Paul Sabatier, CIRIMAT UMR CNRS 5085, 118 route de Narbonne, 31062 Toulouse, France

"| Réseau sur le Stockage Electrochimique de l'Energie (RS2E), FR CNRS 3459, France

\section{Supporting Information}

I. Experimental procedures and simulation method

II. Data processing

III. Additional Figures

S5-S10

IV. References

$\mathrm{S} 10$ 


\section{Experimental procedures and characterization conditions}

1. Transfer of graphene onto Au-coated quartz crystal

BioLogic 1-in.-diameter Au-coated quartz crystals (oscillating frequency, $f_{0}, 5 \mathrm{MHz}$ ) were coated by single layer graphene (SLG). The transfer process of SLG is shown in Figure S1. Firstly, the graphene (G) films were obtained by chemical vapor deposition (CVD) on copper $(\mathrm{Cu})$ substrate. After etching by $0.1 \mathrm{M}$ of ammonium persulfate $\left(\left(\mathrm{NH}_{4}\right)_{2} \mathrm{~S}_{2} \mathrm{O}_{8}\right)$, the transfer of CVD graphene from $\mathrm{Cu}$ to pressure sensitive adhesive (PSA) films coated polyethylene terephthalate (PET) substrate was carried out, as previously reported with modification. ${ }^{1,2}$ The obtained large area PET-graphene films (PET-G) were used to further assist the transfer of graphene to quartz at around $85^{\circ} \mathrm{C}$ for $30 \mathrm{~min}$. The SLG-coated quartz was dried in an oven at $60^{\circ} \mathrm{C}$ for overnight after the PET films were peeled.

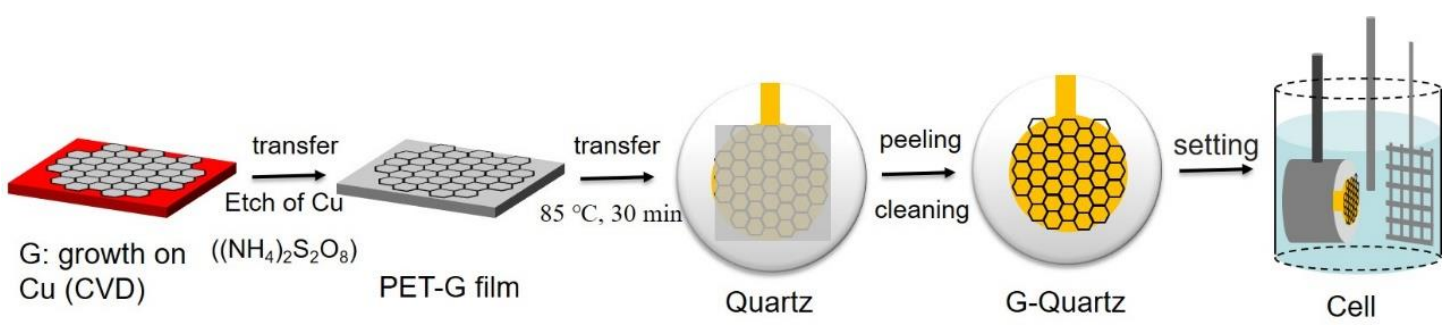

Figure S1. Transfer process of single layer graphene on Au-coated quartz substrate.

2. Electrochemical quartz crystal microbalance setup (EQCM) and EIS measurement

The SLG coated quartz crystal working electrode was placed on a PTFE holder, in which the coated side was orientated toward the reference and counter electrode in a 3-electrode electrochemical cell (Figure S1). The counter electrode was a platinum-coated titanium mesh. The silver wire was used as quasi-reference electrode placed between working and counter electrodes. Three electrodes were set in a glassware and immersed in neat EMITFSI electrolyte. All the EQCM electrochemical measurements were carried out with a Maxtek RQCM system combined with Autolab PGSTAT101 for simultaneous EQCM and electrochemical measurements. Electrochemical impedance spectroscopy (EIS) measurements were carried out with a VMP3 electrochemical working station (Biologic, S.A.). The frequency range studied was $100 \mathrm{kHz}$ to $50 \mathrm{mHz}$, and the measurements were made at different potentials in the range of -0.7 to $0.9 \mathrm{~V}$ versus an $\mathrm{Ag}$ reference electrode. The DV signal amplitude applied was $10 \mathrm{mV}$.

3. Physical characterizations

Graphene characterizations were performed by atomic force microscopy (AFM, Veeco Nanoscope Icon, US), Raman (Labram HR800, $532 \mathrm{~nm}$ laser, France) and optical microscopy (JEOL JSM6400, Japan) for surface and structure analysis.

\section{Simulation method}

Classical molecular dynamics (MD) simulation was performed by using Forcite program based on the Materials Studio. The MD thermal relaxation contains four steps. Step I is geometry optimization of pre-built structure containing random EMI-TFSI configuration 
generated by Amorphous Cell program in Materials studio. Step II is a $3 \mathrm{~ns}$ MD thermal relaxation under NVT ensemble. Step III is a $1 \mathrm{~ns}$ MD annealing process from $300 \mathrm{~K}$ to $1 \mathrm{~K}$ to gradually freeze the structure. Step IV is also a geometry optimization to get the final structure. The length of OA and OB lattice vector is 29.3124 and 29.6114 angstrom and fits the lattice of graphene. The length of OC lattice vector is 100 Angstrom, which is large enough to build the layer in the vacuum. There are $41 \mathrm{EMI}^{+}$and $41 \mathrm{TFSI}^{-}$ions in our simulation model (corresponding to the concentration) and the SLG electrode contains 336 carbon atoms. The carbon atoms in SLG electrode were fixed in all relaxation processes. The ultra-fine quality preset was adopted for geometry optimization and energy calculation. The electrostatic and van der Waals summation was calculated by Ewald method. It should be noted that this model is not suitable to predict the cluster formation during charging process because of the difference between the vacuum environment of ions in modelling and the liquid electrolyte in real measurement.

\section{Data processing}

For the EQCM, the electrode mass change was calculated using Sauerbrey equation: $\Delta \mathrm{m}=$ $-\mathrm{C}_{\mathrm{f}} \cdot \Delta \mathrm{f}$, where $\Delta \mathrm{m}$ is the change of mass of the coating and $\mathrm{C}_{\mathrm{f}}$ is the sensitivity factor of the crystal. The sensitivity factor of the coated quartz was obtained by performing a copper deposition experiment conducted in $0.1 \mathrm{M} \mathrm{CuSO}_{4}$ mixed with $1 \mathrm{M} \mathrm{H}_{2} \mathrm{SO}_{4}$ by applying a constant current of $5 \mathrm{~mA}$ for 120 seconds. In this experiment, the $\mathrm{C}_{\mathrm{f}}$ was calculated to be 27.7 $\mathrm{ng} \cdot \mathrm{Hz}^{-1}$ (or $21.8 \mathrm{ng} \cdot \mathrm{Hz}^{-1} \cdot \mathrm{cm}^{-2}$ taking into account the Au crystal electrode surface of $1.27 \mathrm{~cm}^{2}$ ). For consistent results, few cycles were run before starting EQCM measurements, to start from stable, reproducible electrochemical signatures.

For impedance results, real part $C^{\prime}(\omega)$ and imaginary part $C^{\prime \prime}(\omega)$ of the capacitance $(C(\omega))$ were calculated based on the geometric area of the electrode using the following equation:

$$
\begin{aligned}
& C^{\prime}(\omega)=\frac{-Z^{\prime \prime}(\omega)}{2 \pi f|Z(\omega)|^{2}}, \\
& C^{\prime \prime}(\omega)=\frac{Z^{\prime}(\omega)}{2 \pi f|Z(\omega)|^{2}} \text { and } \\
& C(\omega)=C^{\prime}(\omega)-j C^{\prime \prime}(\omega)
\end{aligned}
$$

where $Z^{\prime}(\omega)$ and $Z^{\prime \prime}(\omega)$ are the real part and imaginary part of the electrochemical impedance $(Z(\omega))$, respectively, defined as $|Z(\omega)|^{2}=Z^{\prime}(\omega)^{2}+Z^{\prime \prime}(\omega)^{2}$, and $f$ is the frequency. We obtained the varied $C_{E D L}$ of single layer graphene by performing impedance experiments at different potentials, and the potential corresponding to the minimum value of capacitance was determined to be the PZC point, as shown in the reference. ${ }^{3}$

Furthermore, the molecular weight of charged ion species $\left(M_{w}\right)$ involved in positive charging was calculated by using Faraday's law, assuming a 100\% faradic efficiency:

$$
\frac{M_{w}}{n F}=\frac{m}{Q}
$$


where $Q$ is the charge passed in the electrode, $\mathrm{m}$ is the corresponding weight change of the electrode during charging process $(\Delta \mathrm{m}-\Delta \mathrm{Q}$ curve $), \mathrm{n}$ is the valence number of the ion species, and $\mathrm{F}$ is the Faraday constant $(96485 \mathrm{C} / \mathrm{mol})$. Taking the minimum valence number of +1 , the average unit of ion species can be statistically expressed by $\left[\mathrm{EMI}_{\mathrm{x}}, \mathrm{TFSI}_{\mathrm{x}-1}\right]^{+}$:

$$
x M_{E M I}+(x-1) M_{T F S I}=M_{w}
$$

where $x$ is the average molar number, $M_{E M I}$ and $M_{T F S I}$ are the molar mass of $\mathrm{EMI}^{+}$and $\mathrm{TFSI}^{-}$, respectively. 


\section{Additional Figures}

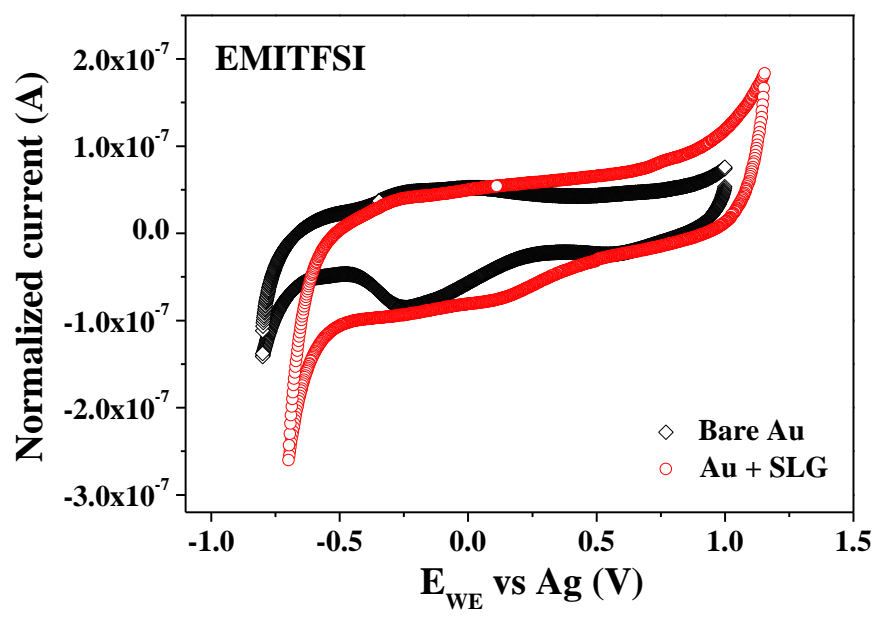

Figure S2. Comparison of CV response of bare gold electrode and single layer graphene coated electrode in EMI-TFSI at $20 \mathrm{mV} \mathrm{s}^{-1}$. The different CV shapes from two samples confirms the presence of SLG before EQCM measurements. When the potential was higher than $1.0 \mathrm{~V}$ (vs Ag) or lower than $-0.5 \mathrm{~V}$ (vs Ag), sharp increased current can be found, which can be ascribed to side reactions from possible trace of water and oxygen (electrolysis). ${ }^{4,5}$

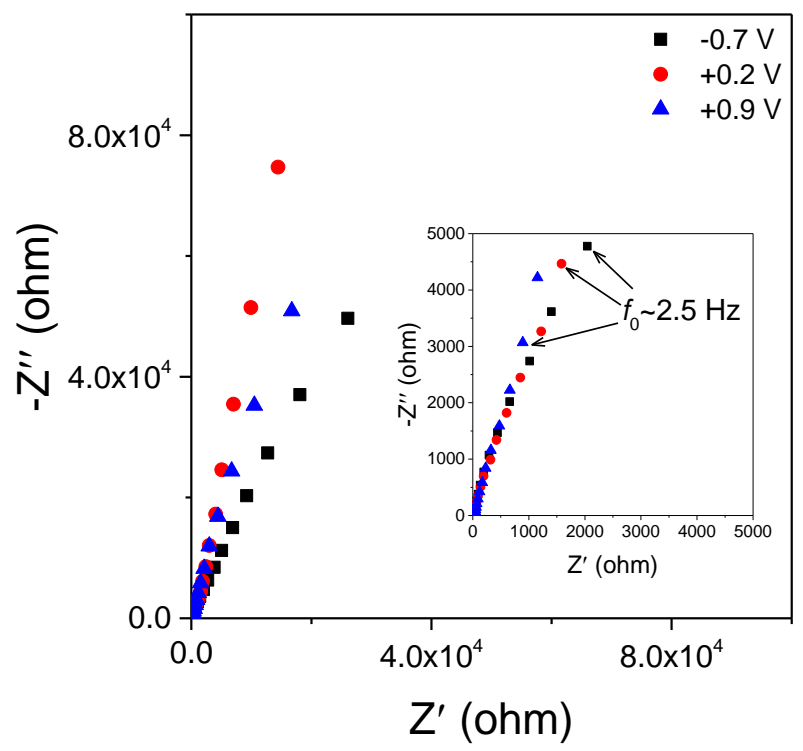

Figure S3. Typical Nyquist plots of single layer graphene measured at different potentials versus Ag reference electrode in neat EMI-TFSI. The potentials were limited in the range of -0.7 to $+0.9 \mathrm{~V}$ in neat EMI-TFSI for EIS measurement. 


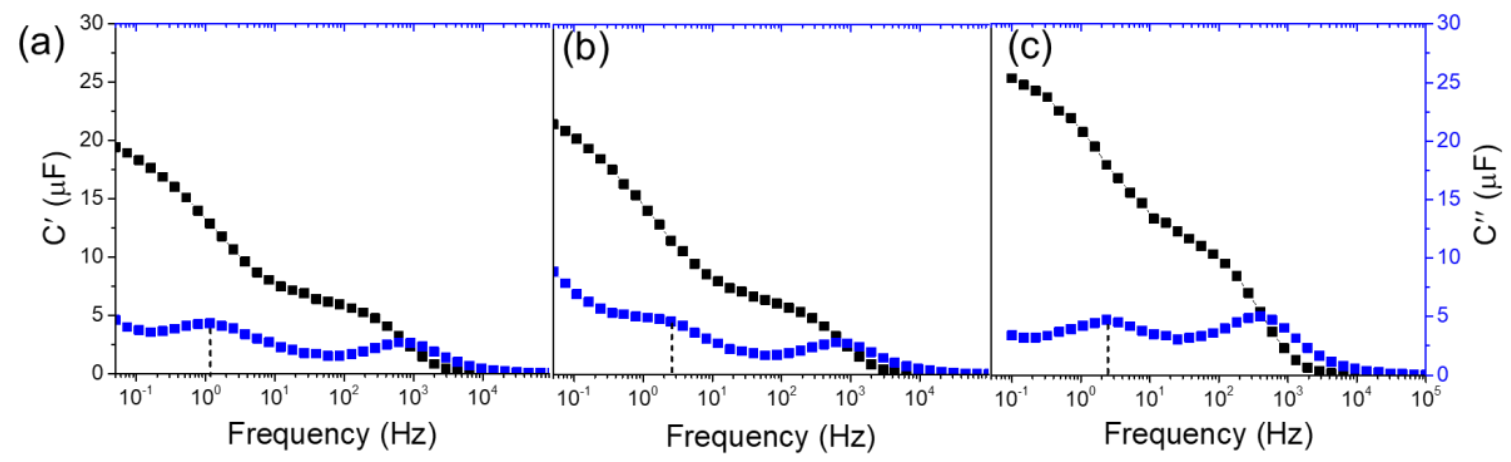

Figure S4. Real parts $C^{\prime}(\omega)$ and imaginary parts $C^{\prime \prime}(\omega)$ of SLG coated quartz electrode in neat EMI-TFSI electrolyte recorded at several typical potentials of (a): $-0.5 \mathrm{~V},(\mathrm{~b}):-0.2 \mathrm{~V}$ (PZC) and (c): $+0.8 \mathrm{~V}$ vs Ref, respectively.

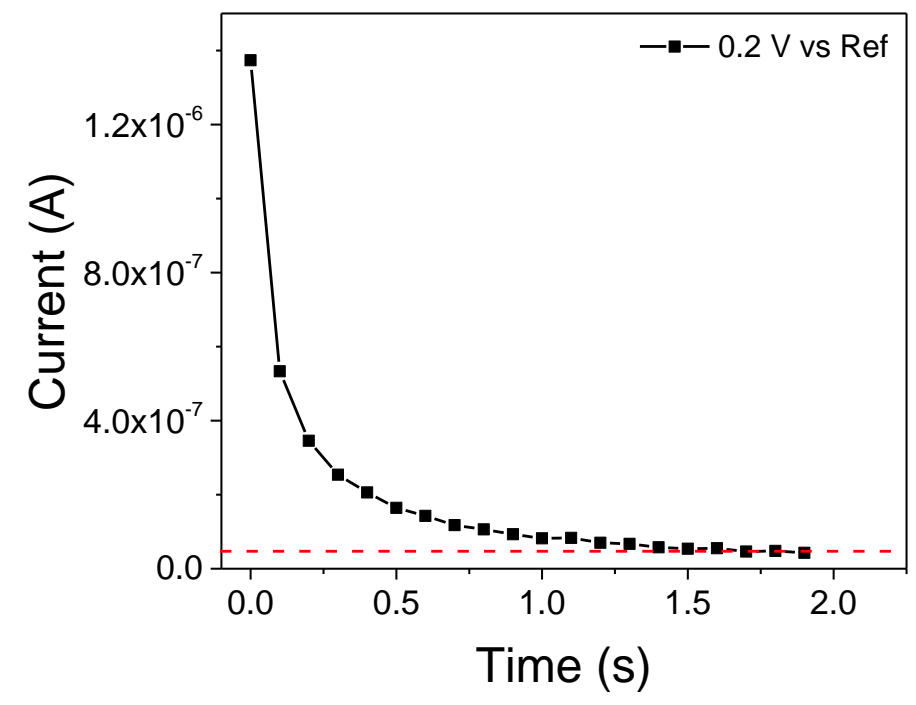

Figure S5. I-t response at a constant potential of $+0.2 \mathrm{~V}$ vs Ref. A constant leakage current of $0.05 \mu \mathrm{A}$ was measured, as indicated by the dotted line parallel to the $\mathrm{X}$-axis. ${ }^{6}$ This result shows that the graphene in RTIL electrolyte cannot be considered as an ideal capacitor but has to be represented by a capacitance $(\mathrm{C})$ in combination with a leakage resistance (Rl) in parallel. The residual, constant leakage current is not associated with the double layer charging, but with redox process from the presence of impurities or/and defects. As a result, the capacitance calculated from EIS in the low frequency range includes a contribution from these minor parasitic redox reactions, leading to a small increase of $\mathrm{C}$ at low frequency. Thus the determination of $\mathrm{C}$ from the time constant is more accurate. 

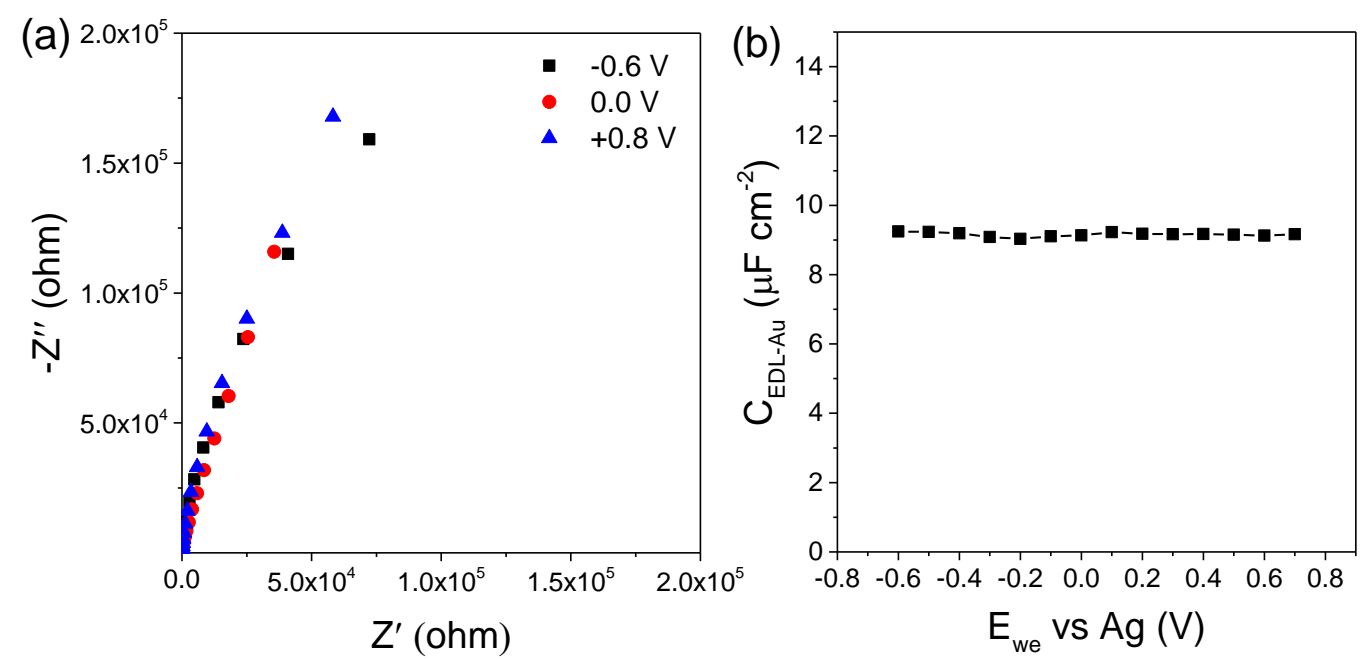

Figure S6. (a) Typical Nyquist plots of pure Au-coated quartz substrate at various potentials

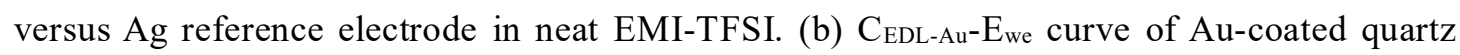
substrate in neat EMI-TFSI. The measured double-layer capacitance was $9.1 \mathrm{uF} \mathrm{cm}^{-2}$ and varied by $2 \%$ within the gate voltage window.

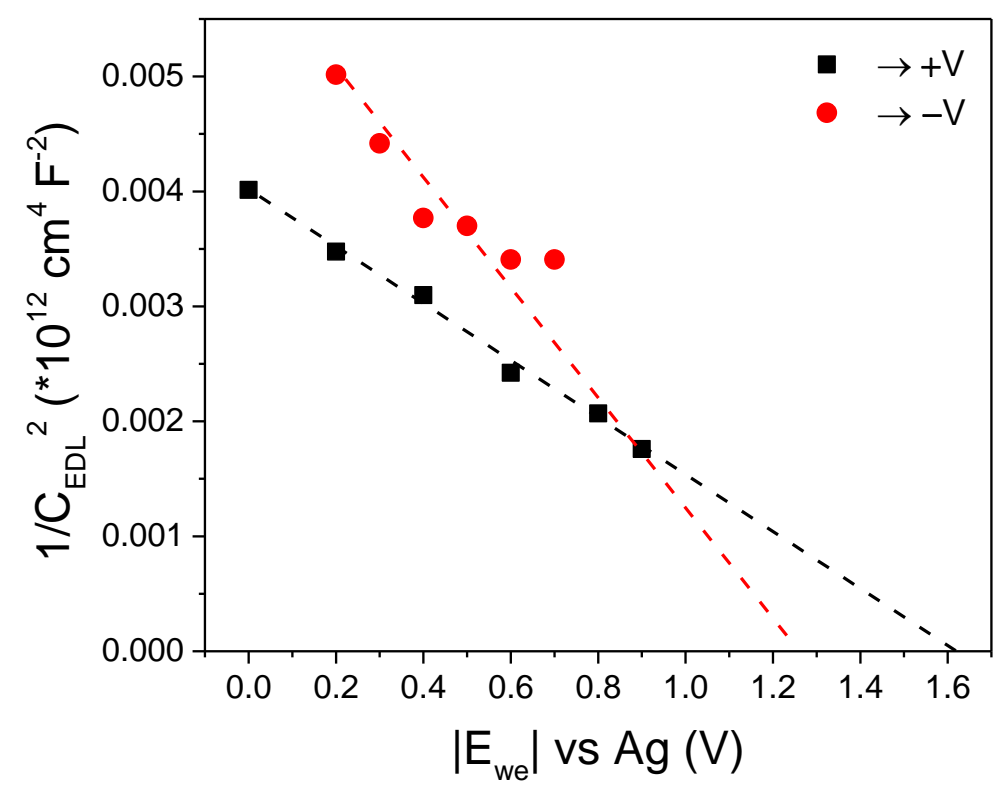

Figure S7. $1 / \mathrm{C}_{\mathrm{EDL}-\mathrm{SLG}}{ }^{2}$ versus $\left|\mathrm{E}_{\mathrm{we}}\right|$ plot derived from the data of the right and left branches of the measured $\mathrm{C}_{\mathrm{EDL}-\mathrm{SLG}}-\mathrm{E}_{\mathrm{we}}$ curve in Figure $2 \mathrm{~b}$. The curves were found to show roughly negative linear correlation. 


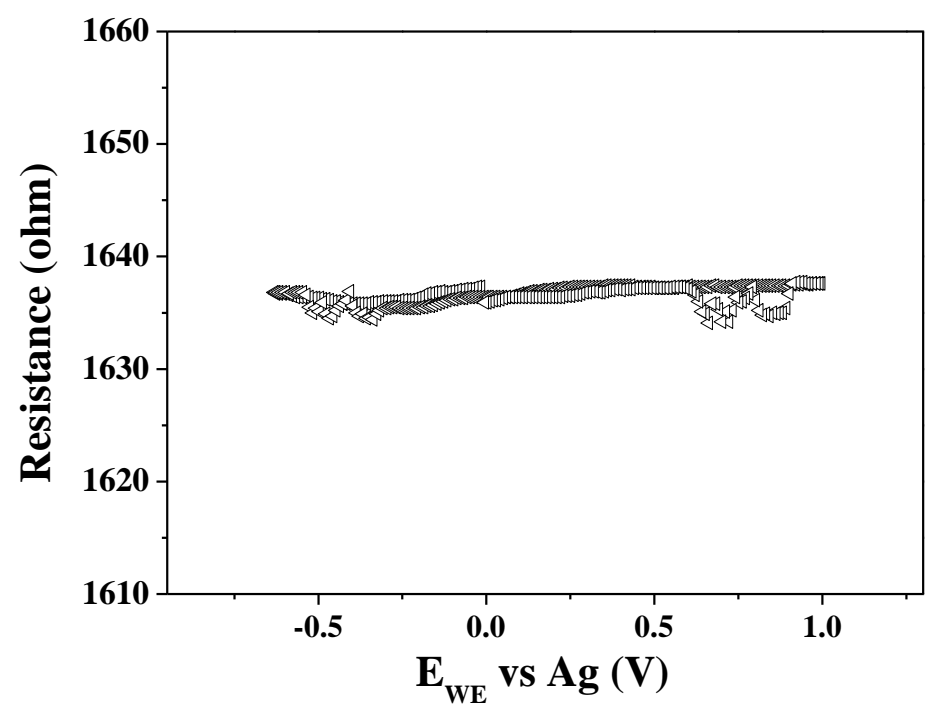

Figure S8. Change of the motional resistance response of single layer graphene on gold substrate in EMI-TFSI at $50 \mathrm{mV} \mathrm{s}^{-1}$. The change of motional resistance was only around $\pm 2 \Omega$, which had no influence to the observed mass change during polarization.
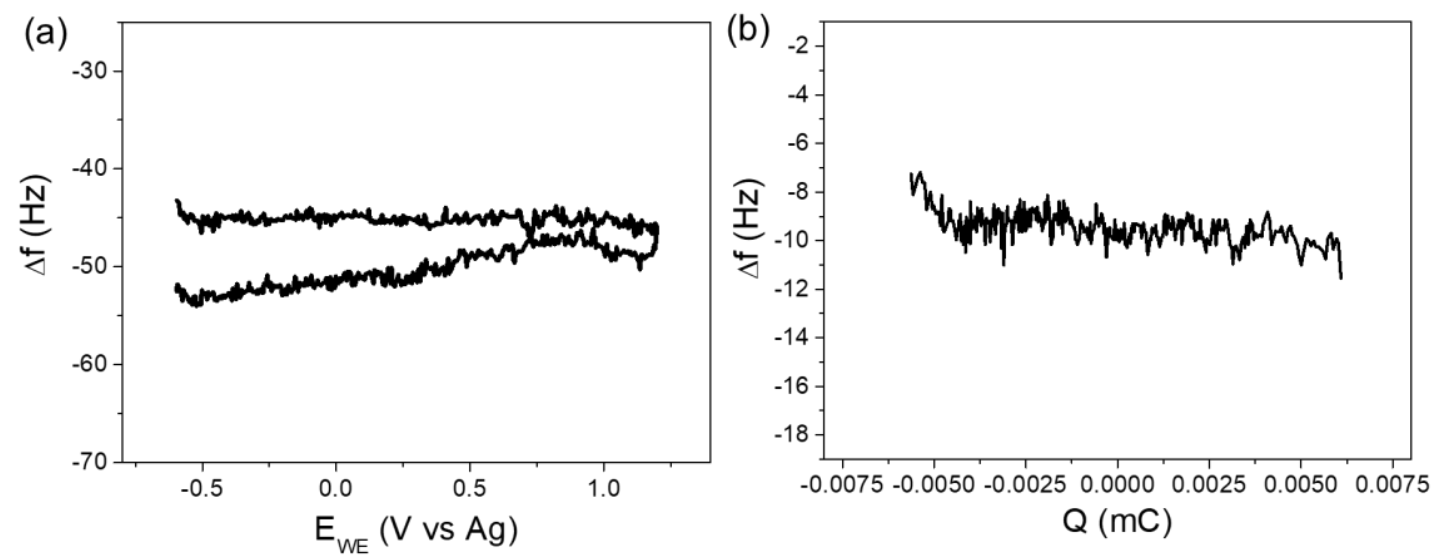

Figure S9. EQCM frequency response ( $\Delta \mathrm{f}$ ) of quartz substrate vs potential (a) and charge (b) during the polarization in neat EMI-TFSI, recorded at $50 \mathrm{mV} \mathrm{s}^{-1}$, respectively. The $\Delta$ f signal does not change significantly during polarization. 
(a)

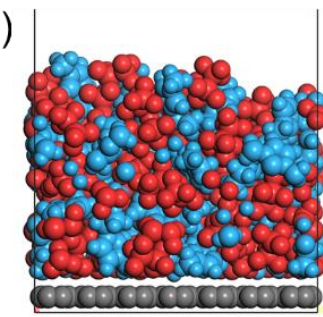

(c)

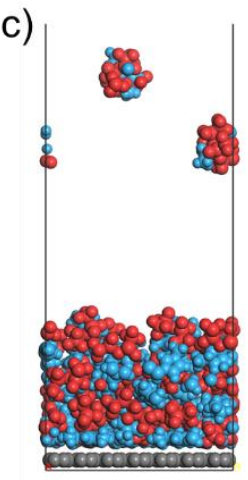

(d) (b)
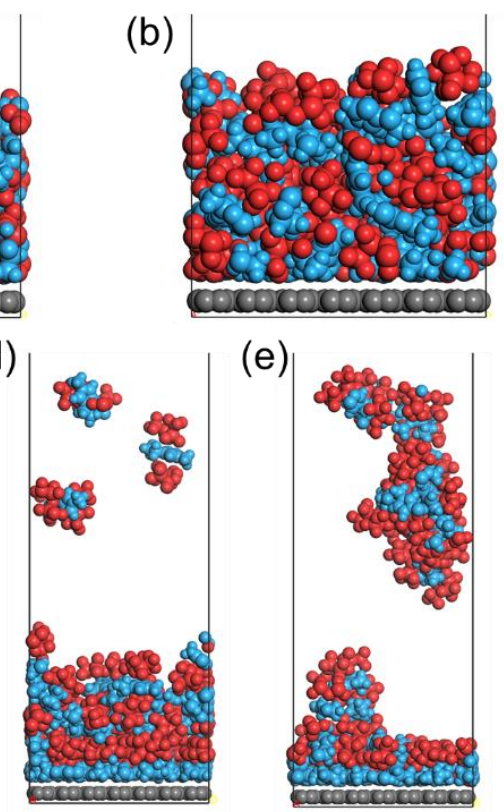

(e)

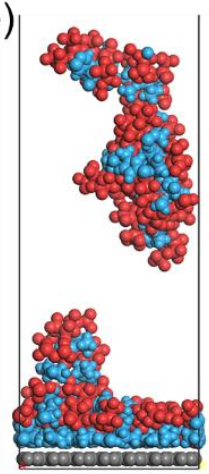

Figure S10. Geometry structures of EMI-TFSI arrangements on graphene electrode with charge of (a) $0.00 \mathrm{e}^{-/ C}$ atom, (b) $0.01 \mathrm{e}^{-/ C}$ atom, (c) $0.02 \mathrm{e}^{-/} / \mathrm{C}$ atom, (d) $0.03 \mathrm{e}^{-/} / \mathrm{C}$ atom, (e) 0.04 $\mathrm{e}^{-} / \mathrm{C}$ atom after MD thermal relaxation.

Obviously, we can see that the arrangement of cations and anions on SLG surface varies

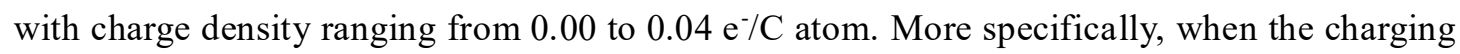
starts for $0.01 \mathrm{e}^{-} / \mathrm{C}$ atom, the ions show the similar adsorption structures as that under neutral condition. When the charge density is increased to $0.03 \mathrm{e}^{-/ C}$ atom (corresponding to $\sim 0.02$ $\mathrm{mC} / \mathrm{cm}^{2}$ ), more $\mathrm{EMI}^{+}$ions are found in the first ion layer contacting SLG; cations and anions are organized in a multi-layered oscillation in the compact adsorption, consistent with previous results. ${ }^{78}$ More importantly, parallel alignment between the $\mathrm{EMI}^{+}$in the first contact layer and graphene surface are also found (Figure S11). In addition, the configuration shown below would narrow the averaged thickness of the double layer and increase the double layer capacitance, as supported by EIS results. From the MD simulations, we can estimate the relative mass of adsorbed ions on the electrode by calculating the mass in $1 \mathrm{~nm}$ thickness on SLG (corresponding to $\sim 2-3$ layers of ions). The mass obtained is 10465, 10450, 10460, 10350 or 9993 relative atomic mass for $0,0.01,0.02,0.03,0.04 \mathrm{e}^{-/ C}$ atom, respectively, supporting the observation that the electrode mass has no significant change during negative charging in the current work. 
(a)
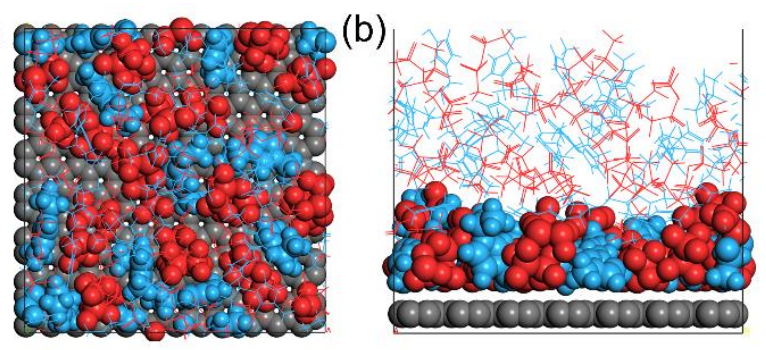

(c)

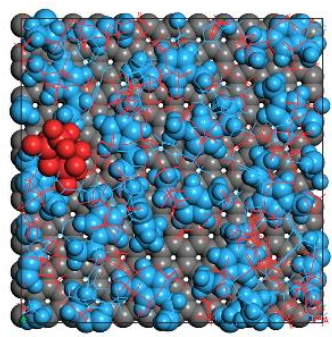

(d)

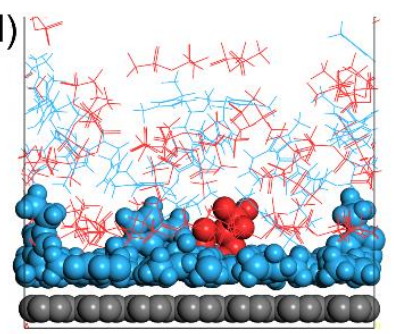

Figure S11. Geometry of first layer of EMI-TFSI ions on SLG electrode in (a) top view and (b) side view for $0.00 \mathrm{e}-/ \mathrm{C}$ atom, and (c) top view and (d) side view of $0.03 \mathrm{e}-/ \mathrm{C}$ atom, respectively.

Principally, the cations are attracted by the graphene surface via $\pi-\pi$ interactions even when no potential is applied, suggesting a mixed cation/anion layering with interfacial iondensified structure, which may be a reason for the fact that applied negative potential does not bring more ions into the compact layer, as discussed above. However, local ion-rearrangement, such as the preferred orientation of cations and the formation of multi-layered oscillation in the compact layer, were also observed, highlighting the critical role of charge-induced nanoscale correlations at SLG/RTIL interface. 


\section{References}

1. Kim, S.; Choi, T.; Lee, B.; Lee, S.; Choi, K.; Park J.; Yoo, J.; Choi, Y.; Ryu, J.; Kim, P.; Hone, J.; and Hong, B. Ultraclean patterned transfer of single-layer graphene by recyclable pressure sensitive adhesive films. Nano lett. 2015, 15, 3236-3240.

2. Ye, J. L.; Tan, H. B.; Wu, S. L.; Ni, K.; Pan, F.; Liu, J.; Tao, Z. C.; Qu, Y.; Ji, H. X.; Simon, P., and Zhu, Y. W. Direct Laser Writing of Graphene Made from Chemical Vapor Deposition for Flexible, Integratable Micro-Supercapacitors with Ultrahigh Power Output. Adv. Mater. 2018, 30, 1801384.

3. Janek, R. P.; Fawcett, W. R.; Ulman, A. Impedance spectroscopy of self-assembled monolayers on $\mathrm{Au}$ (111): evidence for complex double-layer structure in aqueous $\mathrm{NaClO}_{4}$ at the potential of zero charge. J. Phys. Chem. B 1997, 101, 8550-8558.

4. Randström, S.; Montanino, M.; Appetecchi, G. B.; Lagergren, C.; Moreno, A.; Passerini, S. Effect of water and oxygen traces on the cathodic stability of $\mathrm{N}$-alkyl-Nmethylpyrrolidinium bis (trifluoromethanesulfonyl) imide. Electrochim. Acta 2008, 53, 6397-6401.

5. He, M. L.; Fic, K.; Fra, E.; Novák, P.; Berg, E. J. Ageing phenomena in high-voltage aqueous supercapacitors investigated by in situ gas analysis. Energy Environ. Sci. 2016, 9 , 623-633.

6. Prehal, C.; Koczwara, C.; Amenitsch, H.; Presser, V.; Paris, O. Salt concentration and charging velocity determine ion charge storage mechanism in nanoporous supercapacitors. Nat. Commun. 2018, 9, 4145.

7. Uysal, A.; Zhou, H.; Feng, G.; Lee, S. S.; Li, S.; Fenter, P.; Cummings, P. T.; Fulvio, P. F.; Dai, S.; McDonough, J. K.; Gogotsi, Y. Structural origins of potential dependent hysteresis at the electrified graphene/ionic liquid interface. J. Phys. Chem. C 2013, 118, 569-574.

8. Paek, E.; Pak, A. J.; Hwang, G. S. A computational study of the interfacial structure and capacitance of graphene in [BMIM] [PF6] ionic liquid. J. Electrochem. Soc. 2013, 160, A1A10. 